\title{
Hyperstatic Method of Photoelasticity
}

\section{Rajamohan}

Published online: 12 December 2008

(C) Society for Experimental Mechanics 2008

The publisher regrets that the following equation published in the article, "TECHNICAL NOTE: Hyperstatic Method of Photoelasticity," by D. Rajamohan in Experimental Mechanics 48:5, pp. 693-696 has an error. Equation (9) should appear as

$\bar{\varepsilon}^{2}=\frac{4}{3}\left(\varepsilon_{1}^{2}-\varepsilon_{1} \cdot \varepsilon_{2}+\varepsilon_{2}^{2}\right)$

The online version of the original article can be found at http://dx.doi. org/10.1007/s11340-007-9090-4

D. Rajamohan $(\bowtie)$

Mechanical Engineering, Delhi College of Engineering,

H 47 S 2 Near RTO, Tvlvr Nagar,

Madras, Tamilnadu 600 041, India

e-mail: rajamohan.durai@gmail.com 